\title{
Aerosol radiative forcing over land: effect of surface and cloud reflection
}

\author{
S. K. Satheesh \\ Centre for Atmospheric and Oceanic Sciences, Indian Institute of Science, Bangalore - 560012 , India
}

Received: 27 May 2002 - Revised: 28 June 2002 - Accepted: 2 July 2002

\begin{abstract}
It is now clearly understood that atmospheric aerosols have a significant impact on climate due to their important role in modifying the incoming solar and outgoing infrared radiation. The question of whether aerosol cools (negative forcing) or warms (positive forcing) the planet depends on the relative dominance of absorbing aerosols. Recent investigations over the tropical Indian Ocean have shown that, irrespective of the comparatively small percentage contribution in optical depth $(\sim 11 \%)$, soot has an important role in the overall radiative forcing. However, when the amount of absorbing aerosols such as soot are significant, aerosol optical depth and chemical composition are not the only determinants of aerosol climate effects, but the altitude of the aerosol layer and the altitude and type of clouds are also important. In this paper, the aerosol forcing in the presence of clouds and the effect of different surface types (ocean, soil, vegetation, and different combinations of soil and vegetation) are examined based on model simulations, demonstrating that aerosol forcing changes sign from negative (cooling) to positive (warming) when reflection from below (either due to land or clouds) is high.
\end{abstract}

Key words. Atmospheric composition and structure (aerosols and particles) History of Geophysics (atmospheric sciences) Hydrology (anthropogenic effects)

\section{Introduction}

Aerosol particles originate from different sources and production mechanisms. To describe a wide range of possible aerosol compositions, Hess et al. (1998) modeled aerosols as various components (such as sulphates, sea-salt, soot, etc.), each of them meant to be representative for a certain origin. These components can be mixed together to form various aerosol mixtures (such as continental clean, marine polluted, etc). Regional consequences of global warming de-

Correspondence to: S. K. Satheesh

(satheesh@caos.iisc.ernet.in) pend critically on the potentially large cooling or warming effect of aerosols (Crutzen and Andreae, 1990; Ramanathan et al., 2001). Aerosols scatter sunlight back to space and cause a regional cooling effect. These aerosols, consisting of sulfates, sea-salt, soot, organic carbon and mineral dust, are produced both naturally and by human activities. Results of numerous global warming models suggest that the aerosol cooling or warming is one of the largest, if not the largest, source of uncertainty in predicting future climate (Hansen et al., 1998). The question of whether aerosols warm or cool the planet depends on its chemical composition and relative contribution of various chemical species, which constitute the aerosol. An aerosol with significant soot content can have a net warming effect. A completely scattering aerosol increases the value of planetary albedo and hence, cools the planet. If the absorbing aerosol (for example, soot) amount is significant, the net effect of aerosol can be a decrease in the values of planetary albedo due to absorption. This means the warming or cooling effect can change from region to region, depending on many factors, such as the relative strengths of various sources and sinks. The aerosols that have a net cooling effect partly offset the warming due to greenhouse gases, and those that have a net warming effect compliment the greenhouse warming. However, when the amount of absorbing aerosols, such as soot, are significant, aerosol optical depth and chemical composition are not the only determinants of aerosol radiative effects, but the altitude of the aerosol layer and the altitude and type of clouds are also important. In this paper, we examine the effect of different surface types (ocean and land) and cloud reflection on the aerosol forcing based on model simulations.

\section{Results}

During the Indian middle atmosphere programme (IMAP), a project was initiated to monitor the aerosol characteristics over the Indian region by setting up multi-wavelength radiometer (MWR) stations at a few selected sites. The pro- 

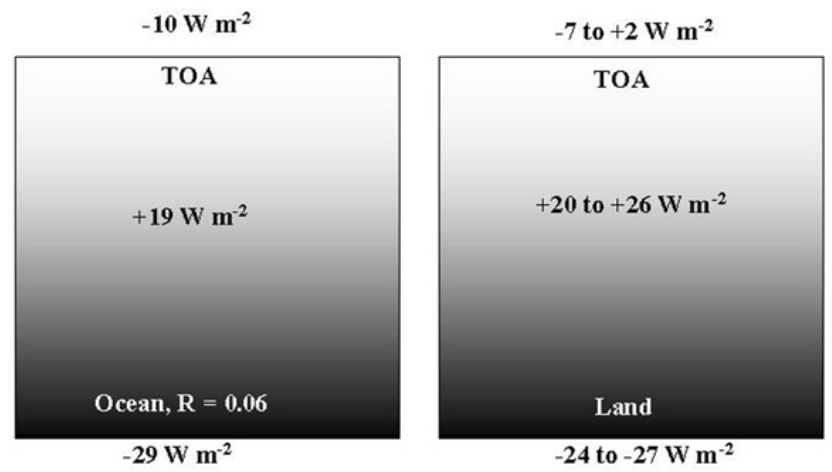

Fig. 1. Comparison of aerosol forcing over the ocean as compared to that over land. The values represent the range of aerosol forcing for different surface types such as soil, vegetation and various combinations of the two.

gramme, which became operational in the late eighties, has been continued after IMAP as part of the Aerosol Climate and Effects (ACE) project of the Indian Space Research Organisation's Geosphere Biosphere programme (ISRO-GBP), with the long-term objective of evolving empirical models of the optical and physical properties of atmospheric aerosols over distinct geographical environments (such as coastal, continental, arid, urban, rural, and industrial) of India. The simultaneous observations at these sites have shown a longterm increasing trend in the aerosol optical depth from 1989 to 1998 (Moorthy et al., 1999). A comparison of the mixed boundary layer aerosol optical depth with the column aerosol optical depth obtained using a solar radiometer has shown that this increasing trend was not confined to the boundary layer alone, but extended to higher altitudes as well (Parameswaran et al., 1998; Moorthy et al., 1999). The increase in aerosol optical depth was about $6 \%$ per year (for the period 1986-1998) at visible wavelengths and about $2.5 \%$ at near-infrared wavelengths.

More than half of the world's population lives in the Asian region. The economical developments in this region during the last decade have resulted in a huge increase in the number of industries and vehicles. The number of vehicles in India has increased from 2 million during 1970 to 38 million during 1998 (Satheesh, 2002). Consequent industrial emissions and anthropogenic activities, such as biomass burning, have created air pollution (aerosols) over most of the Asian region. During the international Indian Ocean Experiment (INDOEX), which was a focussed field experiment in the Indian Ocean designed to assess the significance of anthropogenic aerosols from the Indian and south-Asian subcontinents, the aerosols over the tropical northern Indian Ocean were studied. The aerosol measurements over the tropical Indian Ocean revealed that sulfate and ammonium contributes about $29 \%$ to aerosol optical depth, sea-salt and nitrate about $17 \%$, mineral dust about $15 \%$, and the inferred soot, organics, and fly ash contribute, respectively, $11 \%, 20 \%$ and 8\% (Satheesh et al., 1999). Suresh and Moorthy (2001) reported significant amounts of black carbon over southern
Table 1. Reflectance of different types of surfaces and their combinations

\begin{tabular}{llll}
\hline Surface Type & $0.4-0.7 \mu \mathrm{m}$ & $0.7-1.5 \mu \mathrm{m}$ & $1.5-3.0 \mu \mathrm{m}$ \\
\hline Soil & 0.178 & 0.386 & 0.492 \\
\hline Vegetation & 0.089 & 0.367 & 0.170 \\
\hline 70\% soil; 30\% vegetation & 0.151 & 0.380 & 0.395 \\
\hline 50\% soil; 50\% vegetation & 0.133 & 0.376 & 0.331 \\
\hline 30\% soil; 70\% vegetation & 0.115 & 0.373 & 0.267 \\
\hline
\end{tabular}

India. The above facts indicate that soot may have an important role in the increase in aerosol optical depth during the last decade. This increasing soot may have implications for the regional climate and Indian monsoon (Satheesh and Ramanathan, 2000).

\subsection{Radiative transfer models}

For estimating the aerosol radiative forcing and model simulations, we used a Monte Carlo Radiative Transfer model and a Discrete Ordinate Radiative Transfer mode. A brief description is given here and the details are available elsewhere (Ricchiazzi et al., 1998; Satheesh and Ramanathan, 2000). The Monte Carlo Radiative Transfer (RT) model (designed and developed at the Center for Atmospheric Sciences of the Scripps Institution of Oceanography, San Diego) can be used to estimate the aerosol impact (forcing) on the (SW) broadband fluxes at the surface and the top of the atmosphere (TOA). The band from 0.2 to $4.0 \mu \mathrm{m}$ is divided into 38 narrow bands. The molecular absorption due to water vapor, $\mathrm{CO}_{2}, \mathrm{O}_{2}$, and $\mathrm{O}_{3}$ is taken into account. The zenith angle, dependent ocean albedo (reflectance), is used (Breigleb et al., 1986). Discrete Ordinate Radiative Transfer code is designed and developed by the University of California, Santa Barbara, for the analysis of a wide variety of radiative transfer problems encountered in satellite remote sensing and atmospheric energy budget studies (Ricchiazzi et al., 1998). The program is based on a collection of highly developed and reliable physical models, which have been developed by the atmospheric science community over the past few decades. The discrete ordinate method provides a numerically stable algorithm to solve the equations of plane-parallel radiative transfer in a vertically inhomogeneous atmosphere. The intensity of both scattered and thermally emitted radiation can be computed at different heights and directions. The code is configured to allow for up to 40 atmospheric layers and 16 radiation streams (16 zenith angles and 16 azimuthal modes).

\subsection{Effect of surface reflection on radiative forcing}

Inclusion of measured aerosol properties in the radiative transfer model have shown that the presence of aerosols over the tropical Indian Ocean increases the top of the atmosphere's reflected radiation by $\sim 10 \mathrm{~W} \mathrm{~m}^{-2}$ and decreases the 
Table 2. Effect of different surface types to aerosol forcing $\left(\mathrm{W} \mathrm{m}^{-2}\right)$

\begin{tabular}{lccl}
\hline Surface Type & Surface & TOA & Atmosphere \\
\hline Soil & -24.2 & +2 & +26.2 \\
\hline Vegetation & -27.3 & -7 & +20.3 \\
\hline $70 \%$ soil; 30\% vegetation & -25.4 & -2 & +23.4 \\
\hline $50 \%$ soil; 50\% vegetation & -25.6 & -4 & +21.6 \\
\hline $30 \%$ soil; 70\% vegetation & -26.4 & -5 & +21.4 \\
\hline
\end{tabular}

surface's reaching radiation by $\sim 29 \mathrm{~W} \mathrm{~m}^{-2}$ (Satheesh and Ramanathan, 2000). The estimation of the radiative effects of individual aerosol species have shown that even though soot contributes only $11 \%$ to the aerosol optical depth, it has a very significant role in the forcing due to its absorption properties. Soot contributes about $35 \%$ of the total reduction of solar radiation at the surface (Podgorny et al., 2000; Satheesh and Ramanathan, 2000). This is over the ocean, where surface reflection is very low $(\sim 6 \%)$. If the same aerosol system were present over the land, the effect of soot absorption would be significantly larger because the reflectance of the land is much higher and the radiation reflected from the surface below would interact with the medium (aerosol) again.

A sensitivity study is shown in Fig. 1, where a spectral dependent surface reflectance (corresponding to each wavelength band in the RT model) was used for land. It was assumed that both soil and vegetation constitute land surface. The spectral reflectance of soil and vegetation used are given in Table 1 . Three cases were examined. In the first case, land was assumed to be a combination of $70 \%$ soil and $30 \%$ vegetation coverage. In the second case, it was mixed equally and in the third case, it was $30 \%$ soil and $70 \%$ vegetation. In each case, surface reflection was estimated as the area weighted average of the individual reflectance (Table 1). Over land, the presence of a similar aerosol system, such as reported in Satheesh et al. (1999), would have a surface forcing in the range of -24 to $-27 \mathrm{~W} \mathrm{~m}^{-2}$ and top of the atmosphere forcing in the range of -7 to $+2 \mathrm{~W} \mathrm{~m}^{-2}$, resulting in an atmospheric absorption in the range of +20 to $+26 \mathrm{~W} \mathrm{~m}^{-2}$. This range of values represents all of the above three cases. The detailed analysis of the aerosol forcing in the three cases of surface reflection is given in Table 2. Thus, over land, the presence of absorbing aerosols have a much more significant impact than over the ocean. Also, due to the high surface reflection (in the case of soil), the aerosol forcing at the top of the atmosphere has changed sign from negative to positive (Table 2). In addition, land responds much more quickly to the changes in ground reaching solar flux compared to the ocean due to its low heat capacities and hence, soot over land has many more implications than the similar amount of soot over the ocean.
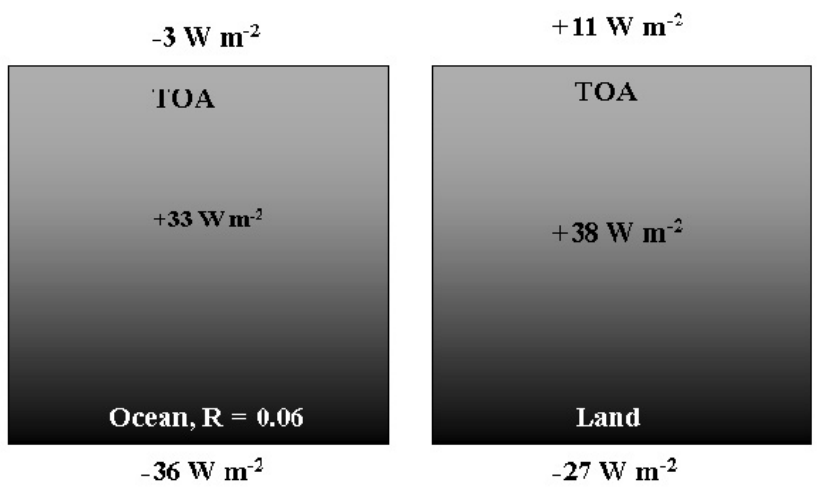

Fig. 2. The effect of doubling the soot contribution to aerosol optical depth and to aerosol radiative forcing. Here the land was assumed as a combination of $70 \%$ soil and $30 \%$ vegetation.

From extensive measurements of soot and size-segregated aerosol concentration over Bangalore $\left(13^{\circ} \mathrm{N}, 77^{\circ} \mathrm{E}\right)$, an urban site in India, Suresh Babu et al. (2002) reported that soot contributes to about $12 \%$ of the total aerosol mass. In INDOEX measurements, as reported in Satheesh et al. (1999), soot contribution to total aerosol mass was only $7 \%$. In the aerosol model of Hess et al. (1998), soot contribution to total aerosol mass for an urban site was $\sim 7.8 \%$. This indicates the presence of high levels of soot over the Indian region. To examine the impact of an increased concentration of soot on the climate, soot contribution to the aerosol optical depth was doubled in the aerosol system (model). The results show that this aerosol system (absorbing) over the ocean (with an optical depth of 0.4 and soot contributing $\sim 22 \%$ to the aerosol optical depth) would reduce the surface reaching solar flux by $\sim 36 \mathrm{~W} \mathrm{~m}^{-2}$ and increases the top of the atmosphere radiation by $\sim 3 \mathrm{~W} \mathrm{~m}^{-2}$, resulting in an atmospheric absorption of $\sim 33 \mathrm{~W} \mathrm{~m}^{-2}$ (Fig. 2). The same aerosol system over land (in the case of $70 \%$ soil and $30 \%$ vegetation) would produce a TOA forcing of $+11 \mathrm{~W} \mathrm{~m}^{-2}$ (Fig. 2) and an atmospheric absorption of $\sim 38 \mathrm{~W} \mathrm{~m}^{-2}$ (Fig. 2). This aerosol heating over the ocean translates to an atmospheric heating of $\sim 0.9^{\circ} \mathrm{K} /$ day, whereas that over land translates to an atmospheric heating of $\sim 1.1^{\circ} \mathrm{K} /$ day. The above results show clearly the adverse effects of an increased soot concentration.

\subsection{Effect of cloud reflection on radiative forcing}

The radiative forcing of aerosols strongly depends on the reflectivity of the underlying surface. The non-absorbing aerosols reflect sunlight and have a cooling effect that is greatest over low albedo surfaces and smallest for high albedo surfaces (Heintzenberg et al., 1997). Light absorbing aerosols also scatter light, and their net effect depends on the relative magnitudes of scattering and absorption (single scattering albedo), as well as the albedo of the underlying surface. This means that the knowledge about the altitude in which the aerosols are distributed and the presence of aerosol layers above the ground is very important. Aerosol 

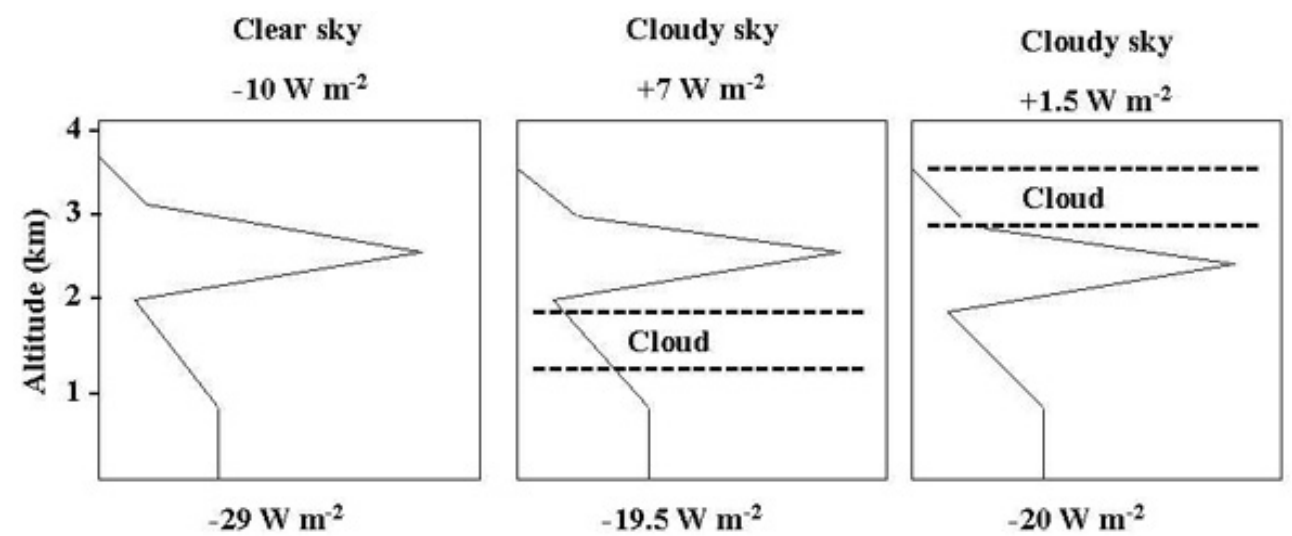

Fig. 3. Effect of cloud reflection on the aerosol radiative forcing. The dotted line represents the cloud layer and the solid line shows a vertical profile of aerosol concentration. The details of cloud properties used are given in the text.

optical depth and chemical composition are not the only determinants of the aerosol effect on the climate. The vertical distribution of aerosol and the type and height of clouds are equally important (Heintzenberg et al., 1997). For a given aerosol optical depth, the aerosol distributed near the surface and that distributed as a layer above the ground have entirely different climate effects. For example, an aerosol layer over the ocean, above a thin sheet of stratocumulus cloud, has an entirely different climate effect compared to an aerosol layer below the cloud. In the same case, the climate effect would be again different if the clouds were absent. These effects would become amplified in the presence of absorbing aerosols, because the light reflected by clouds interacts with the medium (aerosols) again and results in an increased absorption. Thus, aerosol forcing in the presence of clouds is a difficult problem. It is very difficult to measure directly the effect of aerosols alone in the presence of clouds, if not impossible. One way to estimate the aerosol forcing in the presence of clouds is to incorporate accurate aerosol properties into radiative transfer models and then estimate the forcing in the presence of clouds. The observed values of clear sky aerosol forcing at TOA is about $-10 \mathrm{~W} \mathrm{~m}^{-2}$ over the tropical Indian Ocean (Satheesh and Ramanathan, 2000). As in the case of reflecting land surface, cloud reflection from below enhances the absorption effect due to multiple interactions of the light beam with the medium. The effect of the presence of clouds on aerosol radiative forcing is shown in Fig. 3. Here, an optically thin stratocumulus layer was assumed. The cloud droplet number distribution was assumed to follow a modified gamma distribution given by

$$
\frac{d N}{d r}=N a r^{\alpha} \exp \left(-\mathrm{Br}^{\gamma}\right)
$$

where $N$ is the number density in $\mathrm{cm}^{-3}, \alpha$ and $\gamma$ are constants that describe the slope of the distribution, $a$ is a normalising constant ensuring that the integral of size distribution over all radii yields $N$. The value of $B$ is given by

$$
B=\frac{\alpha}{\gamma\left(r_{\text {mod }}\right)^{\gamma}},
$$

where $r_{\text {mod }}$ is the mod radius in $\mu \mathrm{m}$. The values of the size distribution parameters, number density and cloud thickness are varied to obtain a cloud layer with the visible optical depth of 4.0 and a visible region albedo of $\sim 0.47$ and near IR albedo of $\sim 0.39$. In the radiative transfer model, spectral cloud albedo (corresponding to each wavelength band) was used. Cloud coverage was assumed at $50 \%$. Thus, the solar radiation reaching the surface consists of the fraction not intercepted by clouds, as well as the fraction transmitted through the clouds. Similarly, radiation reflected back to space consists of the fraction reflected from clouds and the fraction reflected from the surface (in addition to that backscattered by aerosols and air molecules). It can be seen that the presence of clouds can change the sign of the forcing from negative (cooling) to positive (warming) (Fig. 3). The magnitude of this effect depends on the altitude of the aerosol layer with respect to clouds. In the case of clouds below the aerosol layer, the radiation reflected back from the clouds interacts with the aerosol layer again, thus enhancing the absorption effect. In the case of clouds above the aerosol layer, a fraction of the radiation is intercepted by clouds and hence radiation available to interact with aerosols is smaller and hence, the aerosol influence at the TOA is smaller compared to the former case. In both cases, the presence of a cloud layer changes the sign of TOA forcing from negative to positive. At the surface, aerosol forcing is more or less the same in both cases.

\section{Conclusions}

1. When the amount of absorbing aerosols such as soot are significant, aerosol optical depth and chemical composition are not the only determinants of aerosol climate effects, but the altitude of the aerosol layer and the altitude and type of clouds are also important.

2. Depending on the relative altitude of the aerosol layer and the clouds, the aerosol forcing can vary significantly for the same aerosol concentration and composition. 
3. When surface reflection (either from soil or clouds) is high, the aerosol forcing at the top of the atmosphere changes sign from negative to positive.

4. The large amounts of soot over land areas of the Indian subcontinent might influence the regional climate system.

Acknowledgements. The author thanks Prof. K. Kasturirangan, Chairman, Space Commission of India, for supporting this work as part of ISRO-Geosphere Biosphere Programme. Thanks are due to Prof. J. Srinivasan of Centre for Atmospheric \& Oceanic Sciences at Indian Institute of Science, Bangalore and Dr. K. Krishna Moorthy of Space Physics Laboratory, Trivandrum for valuable suggestions and discussion.

Topical Editor J.-P. Duvel thanks a referee for his help in evaluating this paper.

\section{References}

Breigleb, B. P., Minnis, P., Ramanathan, V., and Harrison, E.: Comparison of regional clear-sky albedos inferred from satellite observations and model computations, J. Climate. Appl. Meteorol., 25, 214-226, 1986.

Crutzen, P. J. and Andreae, M. O.: Biomass burning in the tropics: Impact on atmospheric chemistry and biogeochemical cycles, Science, 250, 1669-1678, 1990.

Hansen, J. E., Sato, M., Lacis, A., Ruedy, R., Tegen, I., and Matthews, E.: Climate forcings in the Industrial era, Proc. Natl. Acad. Sci., 12 753-12 758, 1998.

Heintzenberg, J., Charlson, R. J., Clarke, A. D., et al.: Measurements and modeling of aerosol single scattering albedo: Progress, problems and prospects, Beitr. Phys. Atmosph., 70, 249-263, 1997.

Hess, M., Koepke, P., and Schult, I.: Optical properties of aerosols and clouds: The software package OPAC, Bull. Am. Meteorol. Soc., 79, 831-844, 1998.

Intergovernmental Panel on Climate Change, Climate Change 1994: Radiative Forcing of Climate, report to IPCC from the Scientific assessment Group (WGI), Cambridge Univ. Press, New York, 1995.
Jayaraman, A., Lubin, D., Ramachandran, S., Ramanathan, V., Woodbridge, E., Collins, W., and Zalpuri, K. S.: Direct observations of aerosol radiative forcing over the tropical Indian Ocean during the Jan.-Feb. 1996 pre-INDOEX cruise, J. Geophys. Res., 103(D12), 13 827-13 836, 1998.

Moorthy, K. K., Satheesh, S. K., and Krishna Murthy, B. V.: Investigations of Marine Aerosols over Tropical Indian Ocean, J. Geophys. Res., 102, 18 827-18 842, 1997.

Moorthy, Niranjan, K., Narasimhamurthy, B., et al.: Aerosol Climatology over India. 1 - ISRO GBP MWR network and data base, ISRO GBP SR-03-99, 1999.

Parameswaran, K., Rekha Rajan, Vijayakumar, G., et al.: Seasonal and Long term variations in Aerosol Content in the Atmospheric Mixing Region at a Tropical Station in the Arabian Sea Coast, J. Atmos. Sol. Terr. Phys., 60(1), 17-25, 1998.

Podgorny, I. A., Conant, W. C., Ramanathan, V., and Satheesh, S. K.: Aerosol modulation of atmospheric and surface solar heating rates over the Tropical Indian Ocean, Tellus, 52B, 947-958, 2000.

Ramanathan, V., Crutzen, R. J., Lelieveld, J., et al.: The Indian Ocean Experiment: An Integrated analysis of the Climate Forcing and Effects of the Great Indo-Asian haze, J. Geophys. Res., 106, 28 371-28 398, 2001.

Ricchiazzi, P., Yang, S., Gautier, C., and Sowle, D.: SBDART, A research and teaching tool for plane-parellel radiative transfer in the Earth's atmosphere, Bull. Am. Meteorol. Soc., 79, 21012114, 1998.

Satheesh, S. K., Ramanathan, V., Jones, X. L., Lobert, J. M., Podgorny, I. A., Prospero, J. M., Holben, B. N., and Loeb, N. G.: A Model for the natural and anthropogenic aerosols for the tropical Indian ocean derived from Indian ocean Experiment data, J. Geophys. Res., 104, D22, 27 421-27 440, 1999.

Satheesh, S. K. and Ramanathan, V.: Large differences in the tropical aerosol forcing at the top of the atmosphere and Earth's surface, Nature, 405, 60-63, 2000.

Satheesh, S. K.: Aerosols and Climate, Resonance, 7, 48-59, 2002.

Suresh Babu, S. and Krishna Moorthy, K.: Anthropogenic impact on aerosol black carbon mass concentration at a tropical coastal station: A case study, Current Science, 81, 1208-1214, 2001.

Suresh Babu, S., Satheesh, S. K., and Krishna Moorthy, K.: Enhanced Aerosol Radiative Forcing Observed over Land due to Black Carbon, Geophys. Res. Lett., in press, 2002. 